\title{
Conversations with a neurologist
}

\author{
Interview with Dr Christen Shoesmith
}

\author{
Han Yan (Meds 2017), Ramona Neferu (Meds 2018) \\ Faculty Reviewer: Dr Christen Shoesmith, MD, FRCPC (Department of Clinical Neurological Sciences)
}

In the second of three interviews in this issue, we speak to Dr Christen Shoesmith. Dr Shoesmith is a neurologist and the director of the Motor Neuron Diseases Clinic at the London Health Sciences Centre (LHSC). She runs the local clinical research trials in amyotrophic lateral sclerosis (ALS) and also sits on the ALS Canada Scientific Medical Advisory Panel. She is also heavily involved in medical education at the undergraduate, residency, and fellowship levels. She is an Assistant Professor of Neurology at Western University.

UWOMJ: Tell us a bit about yourself, specifically your education, career, and personal interests.

Christen Shoesmith: After studying medicine at the University of Manitoba, I completed my neurology residency and fellowship here in London. I was fortunate enough to stay on staff after fellowship and have been a staff neurologist at LHSC since 2007.

I subspecialize in ALS and am the clinical director at the Motor Neuron Diseases clinic at University Hospital. I also have a particular interest in patients who have neurological symptoms during pregnancy.

Aside from my career, I love spending time with my husband and my two beautiful daughters who are 7 and 5 years old, and I enjoy jogging and yoga.

\section{Why did you choose neurology?}

I came into medical school with an interest in neurology, but was open to other potential specialties, which I explored through observerships. My undergraduate degree was in chemistry and mathematics, so the analytical and algorithmic approaches to neurological problems were very stimulating for me. I also loved the diverse patient population and the thrill of the diagnosis. In neurology, we see patients in their 20s through their 90s with a variety of neurological disorders. In my first two years of medical school, I attended weekly neurology teaching rounds at the adjoining hospital, and had great mentors who helped me grow in my knowledge and exposure to neurology in medical school.

When I started my neurology residency here at Western, I loved everything about the field, but it became clear early on that I had an interest in palliative care. Initially I explored neuro-oncology but I didn't feel it involved sufficient intellectual challenge for me, since the diagnosis is already there when the patient comes into your care. In contrast, working in the field of neuromuscular disease and ALS gave me the thrill of localization and diagnosis, so it was a natural fit. I also had the complete fortune of having Dr Michael Strong as my mentor in my residency and fellowship. He was instrumental in guiding me through my early career opportunities.

\section{Thoughts on ALS ice bucket challenge?}

In the ALS community, everyone is absolutely thrilled that the ice bucket challenge happened. Patients spread awareness about the disease they're living with, and the community has a much broader understanding of how ALS can affect individuals and families.

Some people in the lay media have spoken out against the ice bucket challenge saying that the funds should have been divided more equitably amongst other diseases initiatives. However, ALS rarely has its face in the spotlight or gets large sums of funding, unlike cancer or heart and stroke.

The other negative publicity issue is that people have looked at the financial statements of ALS Canada and assumed that the distribution of the new funds would be made in the same percentages as previously. However the cost of running the organization won't change substantially, so this one-time donation can be diverted directly to patient support services and research.

\section{Is there a memorable case you would like to share?}

The cases that I remember most are the ones where I have made the right diagnosis that other clinicians may have missed. It is rewarding to know that you've spent the time and energy to make a correct diagnosis. The cases where I have made mistakes or where I did not use the best clinical judgment are also memorable, as well as those cases with complex ethical issues. Some patients have displaced their anger on me about their diagnosis of ALS. I've learned to appreciate that patients have different reactions to diagnoses, and a negative reaction doesn't necessarily mean that you've provided bad care to the patient.

What can you do as a neurologist to make sure that you catch the diagnoses others have missed?

It is very important in neurology to take a great history: let the patient tell their story and not put words into their mouth. Listening to all facets of the story is really important so you can hear about the progression of their symptoms over time. Next, unlike a lot of other areas of medicine, a meticulous physical examination in neurology is still extremely important and it is vital to arriving at a correct diagnosis. To put together a diagnosis, it's important to take the time to think about what all the findings mean. You also can't be afraid to revisit a diagnosis, whether it is your own or someone else's.

The field of neurology is still perceived by many to be a field of diagnosis rather than curing. What are your thoughts on this issue?

From an outsider's perspective, there is the perception that neurology is a "diagnose and adios" profession. But neurology has 
absolutely changed-it's a very different field than when I was a medical student. When I started as a medical student, there were very few treatments available for multiple sclerosis (MS). Now, we've got multiple drugs that have substantially improved the course of MS. Inflammatory conditions, stroke, epilepsy, and seizures have also seen tremendous improvements in management.

There are, of course, neurologic disorders that we can't treat yet, but there are huge amounts of research being done and I certainly think that in my lifetime, diseases like ALS will have better treatments. I never tell my patients we can't do anything about their diagnosis. I explain that we can help manage symptoms with pharmacological management and we also direct people to appropriate allied health professionals. For example, getting an ankle foot orthotic for someone that has foot drop, or getting the appropriate therapist to help with dysphagia can make a huge difference in a patient's quality of life.

How much do you interact with neurosurgeons or psychiatrists?

In London we have a Clinical Neurological Sciences department, so neurosurgeons and neurologists are part of the same faculty, sharing the same resources and physical space. At our site, there is likely more academic interaction between neurology and neurosurgery than at centres where the two specialties are under separate departments (medicine and surgery). This model allows for a shared type of care where we can learn from each other.

\section{Where do you see neurology going in the next 20 years or so?}

There will be more treatments for genetic conditions in neurology. A lot of research is being done but right now there aren't specific treatments for most genetic disorders in neurology. As well, we'll be moving more towards more personalized medicine where different genetic factors will predict different treatments for neurological disorders.
From a career perspective, what are the job options and opportunities for neurologists when they finish residency?

Unlike neurosurgery where underemployment is an issue, neurology doesn't have that problem. There are certainly many urban centres and smaller centres like Thunder Bay and Sudbury that are looking for more neurologists. The majority of the positions are not in academic institutions at the moment, but soon many neurologists will be retiring. As well, because the baby boomer population is getting older and neurological disorders are more prevalent with increasing age, there will be an increased need for neurologists over time.

If students are interested in neurology, what should they do to further explore the field?

For students who are interested in neurology, it's worth getting perspectives from different types of practices and many neurologists through observerships and electives. Medical students can also attend Clinical Neurological Sciences grand rounds, as well as neurology lunchtime rounds. The schedule for weekly rounds is available online on the Clinical Neurological Sciences website. Research is sometimes a bonus when applying for neurology residencies, but not necessary. From a preceptor's point of view, the students that are eager to learn in a clinical setting and have a basic knowledge of neurology/neuroanatomy are the ones that are teachable and a good fit for the program. 